\title{
GTX Regimen
}

National Cancer Institute

\section{Source}

National Cancer Institute. GTX Regimen. NCI Thesaurus. Code C64834.

A reg imen consisting of gemcitabine, docetaxel and capecitabine used for the treatment of pancreatic cancer. 